\begin{tabular}{|c|l|}
\hline Title & $\begin{array}{l}\text { Dorso-ventral axis perturbation in goldfish embry os caused by heat- and pressure shock treatments for chromosome set } \\
\text { manipulation }\end{array}$ \\
\hline Author(s) & Y amaha, Etsuro; Otani, Satoshi; Minami, A tsuy oshi; A rai, Katsutoshi \\
\hline Citation & $\begin{array}{l}\text { Fisheries Science, 68(2), 313-319 } \\
\text { https://doi.org/10.1046j.1474 2906.2002.00427.x }\end{array}$ \\
\hline Issue Date & 2002-04 \\
\hline Doc URL & http://hdl.handle.net/2115/35201 \\
\hline Rights & ○ 2002 公益社団法人日本水産学会; $\odot$ 2002 The Japanese Society of Fisheries Science \\
\hline Type & article \\
\hline File Information & arai-63.pdf \\
\hline
\end{tabular}

Instructions for use 


\title{
Dorso-ventral axis perturbation in goldfish embryos caused by heat- and pressure-shock treatments for chromosome set manipulation
}

\author{
Etsuro YAMAHA, ${ }^{1 *}$ SAToshi OTANI, ${ }^{2}$ Atsuyoshi MINAMI ${ }^{2}$ AND Katsutoshi ARAI $^{2}$ \\ ${ }^{1}$ Nanae Fresh-Water Laboratory, Field Science Center for Northern Biosphere, Hokkaido \\ University, Nanae, Hokkaido 041-1105 and ${ }^{2}$ Laboratory of Breeding Sciences, \\ Graduate School of Fisheries Sciences, Hokkaido University, Hakodate, \\ Hokkaido 041-8611, Japan
}

\begin{abstract}
Effects of the chromosome set manipulation on early embryonic development were examined in goldfish and crucian carp. Both pressure- and heat-shock treatments of fertilized eggs induced developmental disorders such as thin blastodisc formation, delay of epiboly, and deficiency of dorso-anterior structures. The longer the treatments were applied to the eggs, the more frequently severe dorsal deficiencies were induced in the embryos. No and reduced signals of goosecoid mRNA, a dorsal mesodermal marker, were observed in embryos treated with heat-shock at $40^{\circ} \mathrm{C}$ for $1 \mathrm{~min}$ at $5 \mathrm{~min}$ postfertilization. These results suggested that such treatments should affect not only the retention of the second polar body, but also the suppression of dorso-ventral differentiation.
\end{abstract}

KEY WORDS: chromosome set manipulation, crucian carp, dorso-ventral differentiation, goldfish, goosecoid, heat shock, polyploidization, pressure shock.

\section{INTRODUCTION}

Chromosome set manipulation has been used to produce mono-sex, sterile and clonal lines of farmed fish. ${ }^{1-3}$ Although such manipulations have resulted frequently in the abnormal development of embryos, the effects of such treatments on embryogenesis have not been studied. Pressure-, heat- or cold-shock treatment is normally used to inhibit the second polar body release or the first cleavage of the egg. Such treatments may alter the form of tubulin in egg cytoplasm, which plays an essential role in segregating chromosome sets to daughter cells, and then results in an increased chromosome set number. ${ }^{4}$ Tubulin filaments in egg, however, have another function in early development. During blastodisc formation, microtubules may transport some cytoplasmic particles and/or factors, which regulate early cellular differentiation of blastomeres, from the vegetal to the

*Corresponding author: Tel: 81-138-65-2344. Fax: 81-138-652239. Email: eyamaha@fsc.hokudai.ac.jp

Received 10 April2001. Accepted 25 September 2001. animal hemisphere of embryos or vice versa. ${ }^{5,6}$ In zebrafish, cold-shock treatment affecting cytoplasmic tubulin filaments gives rise to abnormal development, including perturbation of dorsal-ventral specification. ${ }^{7}$ However, no studies have investigated the side-effects of chromosome set manipulation on the normal process of dorso-ventral specification in developing fish embryos.

In the present study, we studied the effects of pressure- and heat-shock treatments on the early development of fertilized eggs, especially dorsoventral differentiation of goldfish and crucian carp embryos.

\section{MATERIALS AND METHODS}

\section{Fertilization and cultivation}

Goldfish and crucian carp Carassius auratus were reared at the Graduate School of Fisheries Sciences, and Nanae Fish Culture Experimental Station, Hokkaido University. Ovulation was induced by hormonal injection, and artificial 
insemination and fertilization were performed as described elsewhere. ${ }^{8}$ Briefly, inseminated eggs were fertilized in the fertilization solution, which contained $0.2 \%$ urea and $0.25 \% \mathrm{NaCl}$ in tap water. The fertilization solution was exchanged with tap water at approximately $3 \mathrm{~h}$ postfertilization (h.p.f.).

To trigger gynogenetic development, sperm was irradiated with ultraviolet light (UV sperm) and inseminated into mature eggs, following the method described by Onozato and Yamaha ${ }^{9}$ but with slight modifications. Resultant haploid gynogenes were distinguished from diploid ones by their external appearance, collectively described as haploid syndrome which included abnormalities such as small eyes with incomplete pigmentation, dwarfed body size, bent body shape, and edema. Haploidy in embryos displaying these abnormalities is always confirmed by flow cytometry (data not shown).

Eggs treated with high temperature or hydrostatic pressure (as described later) and control eggs were incubated in tap water at $20^{\circ} \mathrm{C}$ for 3 days by exchanging the water and then removing dead eggs daily. Thereafter, the dorso-anterior index (DAI; described later) was scored for all embryos. Haploidy or diploidy was classified based on the external appearance of embryos at 3 days postfertilization (d.p.f.).

\section{Heat-shock and pressure-shock treatments}

Heat-shock treatment was carried out according to Nagoya et al. ${ }^{10}$ with slight modifications. Eggs inseminated with UV sperm or normal sperm were fertilized in platic Petri dishes filled with the fertilization solution. At $5 \mathrm{~min}$ postfertilization (m.p.f.), eggs on the plastic Petri dishes were immersed in warmed $\left(40^{\circ} \mathrm{C}\right)$ fertilization solution for a given duration of between $30 \mathrm{~s}$ and $4 \mathrm{~min}$.

Eggs for pressure-shock treatment were fertilized on glass slides immersed in fertilization solution. The eggs that adhered to the glass slides were inserted into the cell of a French Press apparatus (Otake Works Co. Ltd, Tokyo, Japan) filled with fertilization solution. At 5 m.p.f., $700 \mathrm{~kg} / \mathrm{cm}^{2}$ of hydrostatic pressure was applied for a given duration lasting between $20 \mathrm{~s}$ and $4 \mathrm{~min}$.

\section{Scoring of dorso-anterior index}

The DAI, which has been described previously by Mizuno etal.,11 was used for the relative index of ventralization of treated embryos. The external characteristics of each DAI were as follows: DAI 1 , rotational symmetry (no axis); DAI 2, truncated head; DAI 3, cyclops; DAI 4, joined eyes; DAI 5, normal. This goldfish DAI grading system is analogous to the dorso-anterior index for Xenopus, ${ }^{12}$ with the exception that the non-axial, radialized embryos of goldfish were scored as DAI 1 instead of DAI 0 as in Xenopus.

\section{Whole-mount in situ hybridization}

For in situ hybridization, a part of the treated and control embryos were dechorionated with $0.1 \%$ trypsin (Difco, Detroit, MI, USA) and $0.4 \%$ urea in Ringer's solution (128 mM NaCl, $2.8 \mathrm{mM} \mathrm{KCl}$, $\left.1.8 \mathrm{mM} \mathrm{CaCl}_{2}\right){ }^{13}$ Dechorionated eggs were cultured in a plastic Petri dish coated with $1 \%$ agar and filled with culture Ringer's solution containing $1.6 \%$ albumen. Thereafter, the dechorionated embryos were fixed with $4 \%$ paraformaldehyde fixative at the late blastula to the embryonic shield stage at $4^{\circ} \mathrm{C}$ overnight, and used for in situ hybridization. The developmental schedule for goldfish during pregastrula has been staged previously by Yamaha et al.; ${ }^{15}$ the late blastula and the embryonic shield stage occurred approximately 8 h.p.f. and 15 h.p.f., respectively.

In situ hybridization was carried out as described elsewhere ${ }^{11}$ with antisense RNA probes prepared from a DIG RNA labeling kit (Boehringer Mannheim, Mannheim, Germany). A clone containing the gsc cDNA fragment, which excludes the homeo domain of the zebrafish, was used as the template for transcription. In teleosts, gsc expression during the stage from late blastula to gastrula is essential for the formation of dorsal structures at later stages. ${ }^{12}$

\section{RESULTS}

Embryos that developed from heat shock-treated eggs at 5 m.p.f. displayed thin blastodiscs with poorly developed cytoplasm (Fig. 1). Many large particles were observed in the blastodisc cytoplasm. This blastodisc had an unclear border between the cytoplasm and yolk. Meridional cleavage furrows in treated eggs were shallower than those in normal control eggs. When epiboly began in the control embryos, the blastoderm of embryos developed from treated eggs was a hemispherical mass of blastomeres. The epiboly of treated eggs began later than in the control group. The embryonic shield of treated eggs was obscure in the germ ring at 15 h.p.f.

-At 2 d.p.f., many embryos that were developed from treated eggs showed typical dorso-anterior deficiencies with DAI scores of $1-4$, whereas 
control embryos that developed up to the optic cup stage showed embryonic body formation (Fig. 2). Mortality and frequency of embryos with severe DAI scores increased proportionally with duration of heat-shock treatment (Table 1).
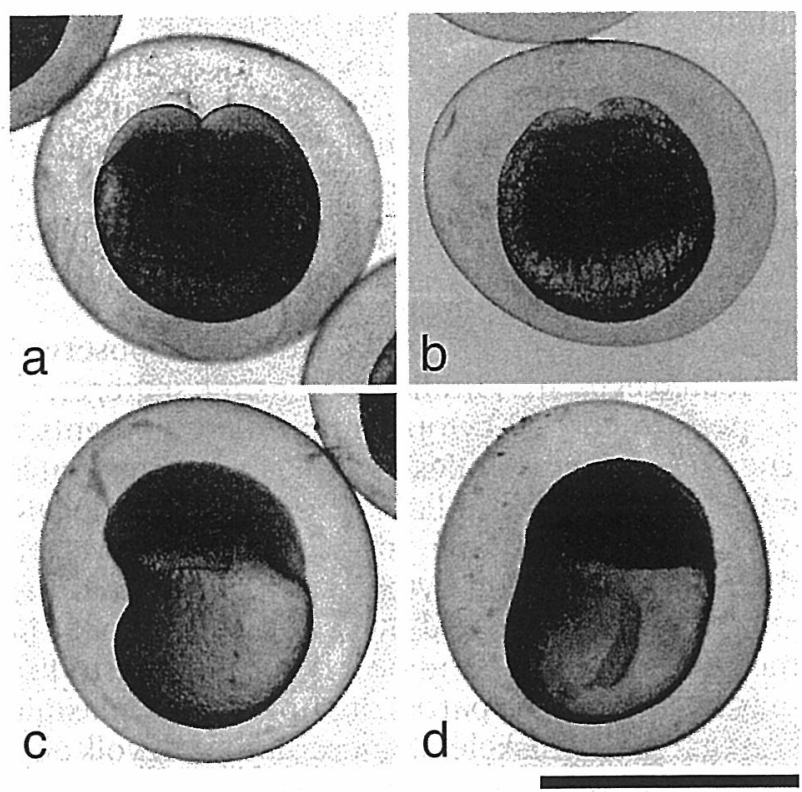

Fig. 1 External appearances of embryos from heat shock-treated eggs at the $(\mathrm{a}, \mathrm{b})$ two-cell and $(\mathrm{c}, \mathrm{d})$ late blastula stage. (a,c) Control embryo. (b,d) Heat shocktreated embryos treated at $5 \mathrm{~min}$ postfertilization. Bar $=1 \mathrm{~mm}$.
Normal diploid gynogenetic embryos were obtained at 3 d.p.f. for all experimental groups that were developed from the eggs of three females (Nos 1-3), activated by UV sperm, and then treated with theat shock (Table 1). When the same heatshock treatment conditions were applied to eight broods from other different females, the frequency of diploid embryos relative to total eggs treated was different between the broods (data not shown). The highest value was $54.5 \%(686 / 1258)$, and the lowest was $0 \%(0 / 1037)$ in the eight broods (average 15.4\%).

In situ hybridization was used to investigate gsc expression in the embryos developed from heat shock-treated eggs of goldfish and crucian carp. In the embryos from eggs that underwent heat shock treatment for $75 \mathrm{~s}$ and $90 \mathrm{~s}$, high percentages of no signals (13.0-74.6\%) and reduced signals of gsc expression (6.8-35.2\%) were observed, whereas only one reduced expression of gsc was observed in the control groups (Fig. 3, Table 2).

Pressure-shock treatment also induced abnormalities that were similar to those observed in embryos developed from heat shock-treated eggs (Table 3). The incidence of mortality and the frequency of embryos with severe DAI scores both increased with increased duration of pressureshock treatment. When the eggs were fertilized by normal sperm and then treated with pressure shock, we observed abnormalities similar to those observed in gynogenetically-induced embryos.
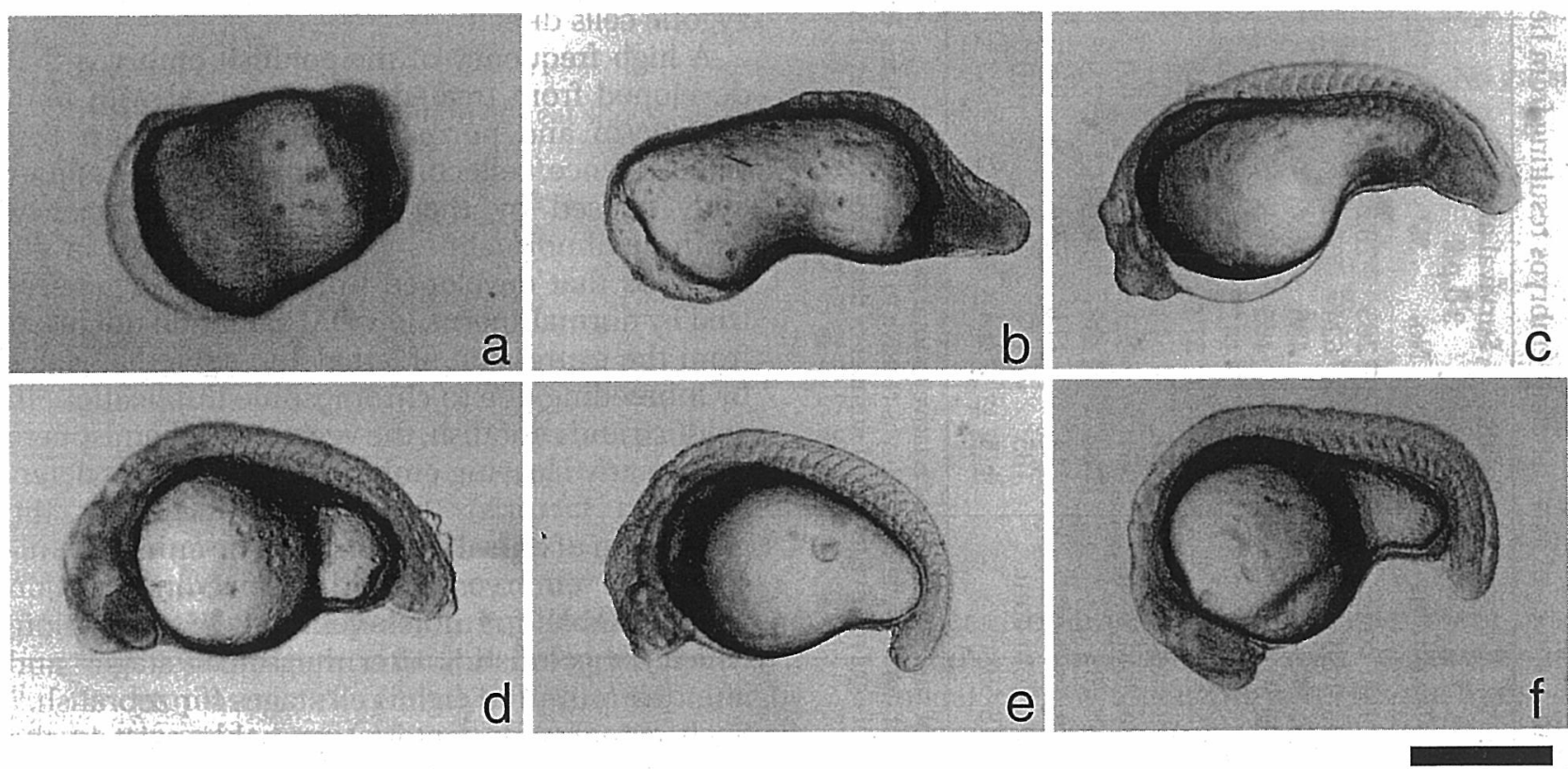

Fig. 2 External appearances of dorso-anterior deficient embryos at 2 days postfertilization developed from heat-shock treatment at 5 min postfertilization. (a) Dorso-anterior index (DAI) 1; (b) DAI 2; (c) DAI 3; (d) DAI 4; and (e) DAI 5 embryos from heat-shocked eggs. (f) Control embryo. Bar $=0.5 \mathrm{~mm}$. 


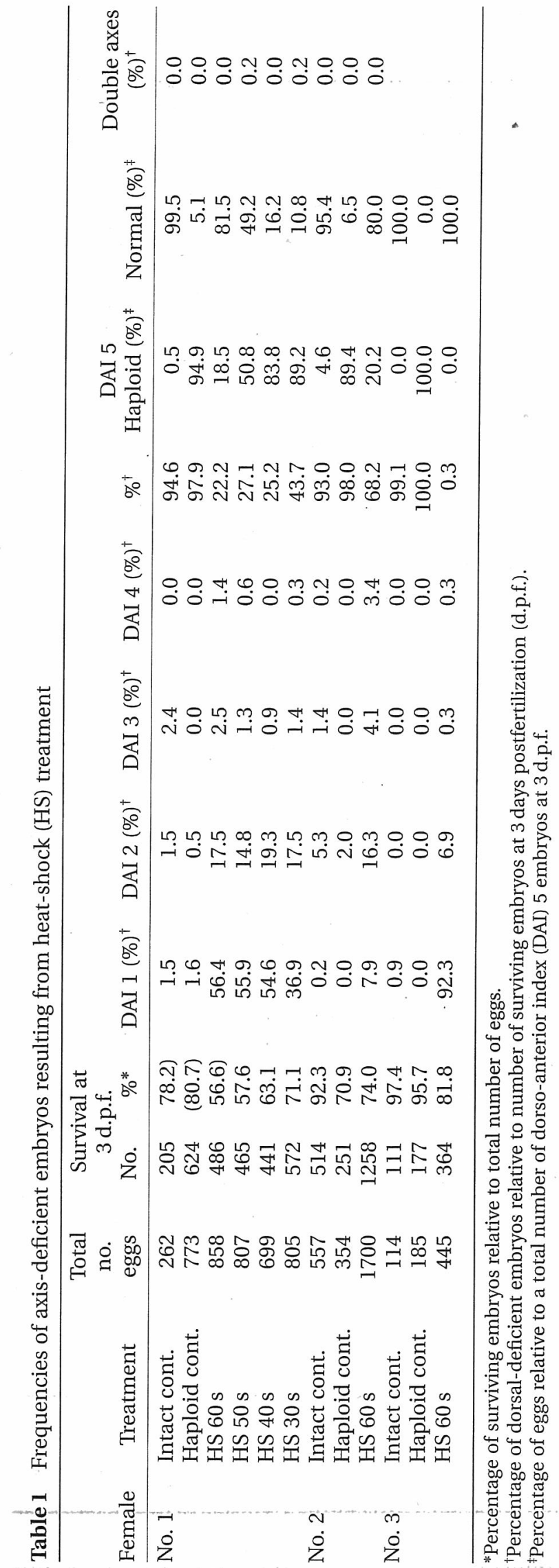

\section{DISCUSSION}

Both heat-shock and hydrostatic-pressure treatments apparently caused developmental disorders in goldfish embryos. Such treatments have been reported previously to cause disruptions in microtubules. ${ }^{4}$ In the present study, because normal diploid embryos were obtained in the treated eggs fertilized with UV sperm, these treatments were effective in disrupting spindle fibers at the second maturation division. Therefore, the developmental disorders observed in the present study may have to do with the microtubules distributed in the zygote just after fertilization.

Poor formation of the blastodisc was observed in treated eggs. In zebrafish, ooplasmic segregation is performed by cytoplasmic microfilaments. ${ }^{16}$ The role of microtubules in ooplasm segregation is unclear because bundles of microtubules locate only the vegetal pole in zebrafish. ${ }^{17}$ However, the transportation of cytoplasmic particles to the animal pole is inhibited by colcemid treatment in medaka. ${ }^{5}$ Therefore, the poorly formed blastodisc may result from the partial disruption of ooplasmic segregation. Cytoskeletal microtubules in yolk also play an essential role in epiboly. ${ }^{18}$ In zebrafish, the disruption of yolk microtubules inhibits epiboly and causes the accumulation of blastoderm cells at the animal pole. ${ }^{7}$ In the present study, heatshocked eggs showed delayed epiboly, but epiboly proceeded in most treated embryos, suggesting that high-temperature and pressure treatments would affect the cytoskeletal microtubules of zygotic cells directly.

A high frequency of the goldfish embryos that developed from treated eggs fertilized with both UV sperm and normal sperm showed low DAI values; hence, reflecting the abnormalities apparently caused by the dorso-anterior deficiency. As these abnormalities were also observed in the embryos that developed from treated eggs fertilized by normal sperm, low DAI values do not result from the expression of lethal and sublethal genes by inbreeding due to chromosome duplication. In goldfish and zebrafish, the vegetal yolk hemisphere of the early cleaving embryos contains axis determination factor(s) that are necessary for the generation of dorsal structures. ${ }^{11}$ The animal hemisphere of embryos should receive information indispensable for dorso-ventral axis formation, which for goldfish is after the 16-cell stage ${ }^{11}$ and after the four- and eight-cell stages for zebrafish. ${ }^{19}$ Such cytoplasmic factor(s) probably exist in the upstream of the cascade of dorso-ventral axis formation, leading to the expression of gsc. It is presently unclear whether or not this factor(s) originally localized around the vegetal pole moves 

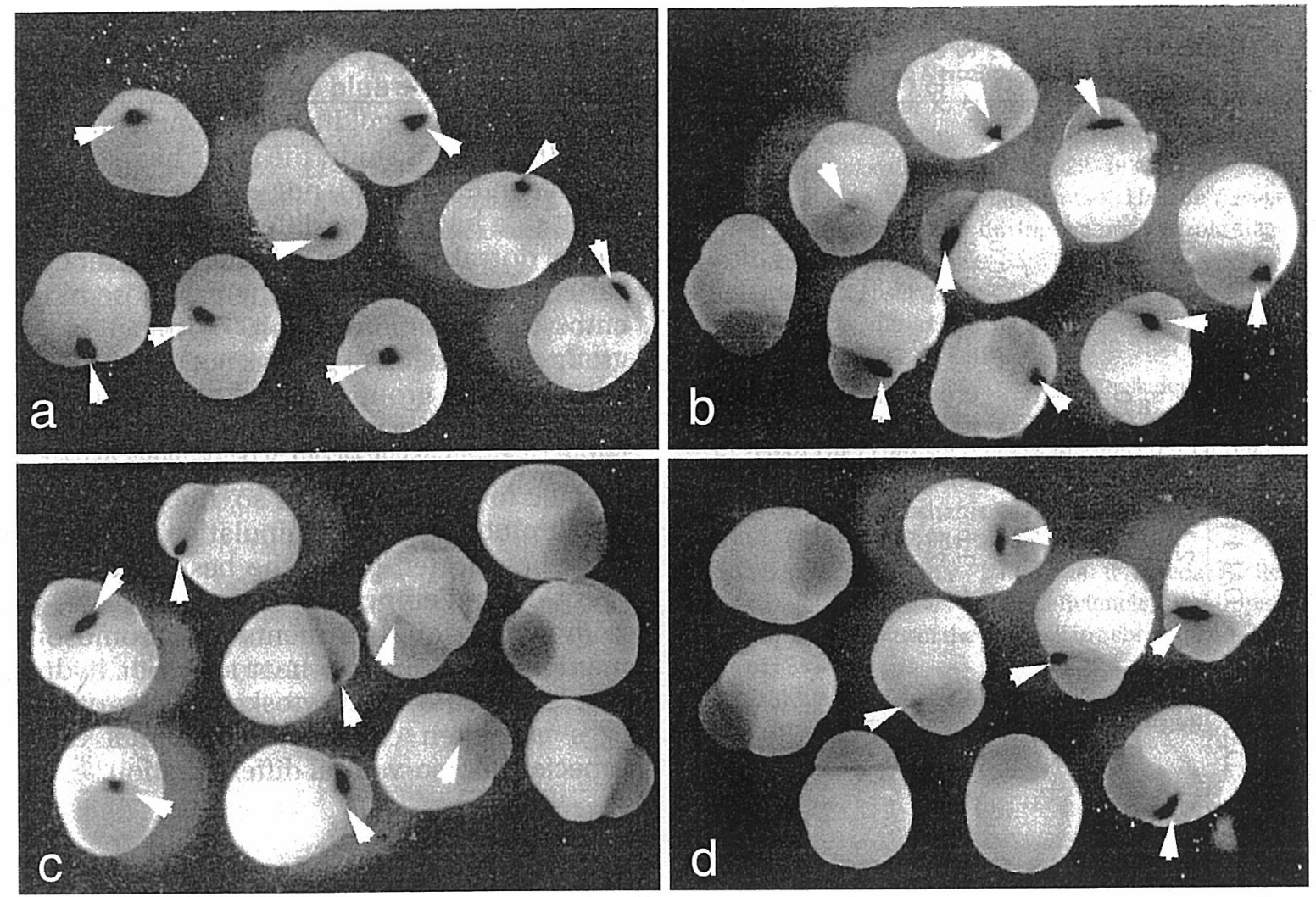

Fig. 3 Expression of goosecoid at $12 \mathrm{~h}$ postfertilization in (a) control embryos and (b-d) heat shock-treated embryos. Embryos were treated at $40^{\circ} \mathrm{C}$ for (b) $30 \mathrm{~s}$; (c) $60 \mathrm{~s}$, and (d) $90 \mathrm{~s}$ at 5 min postfertilization. Bar $=1 \mathrm{~mm}$.

Table 2 Frequencies of embryos expressing normal, reduced and no signal of goosecoid (gsc)

\begin{tabular}{|c|c|c|c|c|c|c|c|c|}
\hline \multirow[b]{2}{*}{ Experiment } & \multirow[b]{2}{*}{ Duration of heat shock (s) } & \multirow[b]{2}{*}{ No. eggs } & \multicolumn{2}{|c|}{$\begin{array}{l}\text { Normal gsc } \\
\text { signal }\end{array}$} & \multicolumn{2}{|c|}{$\begin{array}{l}\text { Reduced gsc } \\
\text { signal }\end{array}$} & \multicolumn{2}{|c|}{ No gsc signal } \\
\hline & & & No. & $\%$ & No. & $\%$ & No. & $\%$ \\
\hline \multirow{5}{*}{$\begin{array}{l}\text { No. } 1 \\
\text { (Goldfish) }\end{array}$} & 0 & 34 & 33 & 97.1 & 1 & 2.9 & 0 & 0.0 \\
\hline & 45 & 48 & 47 & 97.9 & 0 & 0.0 & 1 & 2.1 \\
\hline & 60 & 50 & 46 & 92.0 & 3 & 6.0 & 1 & 2.0 \\
\hline & 75 & 54 & 36 & 66.7 & 11 & 20.4 & 7 & 13.0 \\
\hline & $\cdot 90$ & 54 & 18 & 33.3 & 19 & 35.2 & 17 & 31.5 \\
\hline \multirow{5}{*}{$\begin{array}{l}\text { No. } 2 \\
\quad \text { (Crucian carp) }\end{array}$} & 0 & 62 & 61 & 98.4 & 1 & 1.6 & 0 & 0.0 \\
\hline & 45 & 60 & 50 & 83.3 & 3 & 5.0 & 7 & 11.7 \\
\hline & 60 & 57 & 42 & 73.7 & 4 & 7.0 & 11 & 19.3 \\
\hline & 75 & 58 & 28 & 48.3 & 6 & 10.3 & 24 & 41.4 \\
\hline & 90 & 59 & 11 & 18.6 & 4 & 6.8 & 44 & 74.6 \\
\hline
\end{tabular}

toward the animal pole during the early cleavage stage. Jesuthasan and Strähle have proposed that the cytoplasmic movement, which is mediated by the transient array of microtubules in the yolk cortex, would require axis specification. ${ }^{6}$ Their hypothesis is supported by the observation that the disruption of microtubules soon after fertilization gave rise to the frequent occurrence of dorsal- defective embryos. ${ }^{6}$ Results of the present study of goldfish coincides with their observations. In medaka, the cytoplasmic particles are transported by microtubules from the vegetal pole to the animal pole and vice versa ${ }^{5}$ and, furthermore, the direction of the microtubule array coincides with the future embryonic axis. ${ }^{20}$ These results suggest that the dorsal signal might be transported by 


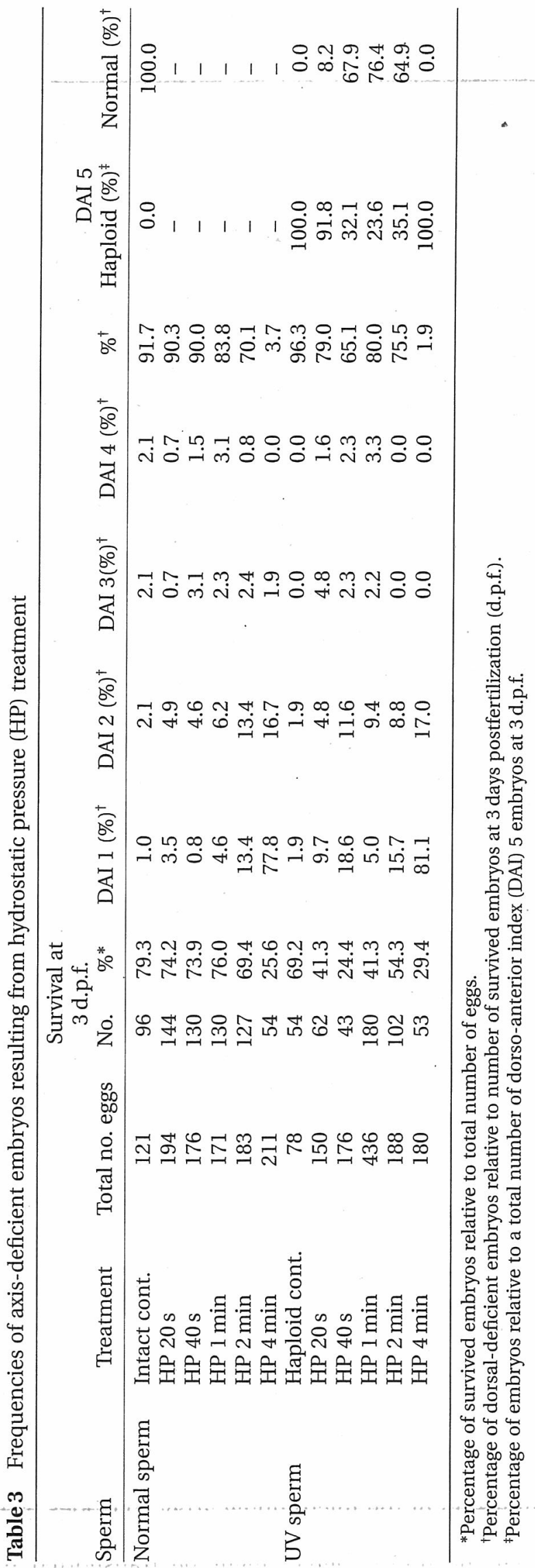

microtubules. Although no conclusive evidence has been obtained yet, chromosome set manipulation treatments would disturb axis specification at the early stage of goldfish development.

In the present study, the same heat-shock treatment conditions did not always give the same result in a batch from different females. Frequencies of dorsal deficiencies in the resultant embryos varied between females, whereas those of normal embryos were more than $90 \%$ intact in control groups. Expression of gsc was not always decreased even in those groups treated with heat shock for $60 \mathrm{~s}$. These results might demonstrate that the sensitivity of eggs to heat-shock treatment might be specific for each brood. If so, the best conditions for chromosome set manipulation for each brood must be selected to get the best yield of normal polyploidized embryos.

In conclusion, treatments for chromosome set manipulation, such as heat shock or hydrostatic pressure, would affect cytoskeletal microtubules in eggs and then cause developmental disorders, especially dorso-ventral differentiation.

\section{ACKNOWLEDGMENTS}

We thank our former members of the Laboratory of Embryology and Genetics, Faculty of Fisheries, Hokkaido University, especially Mr Y Hasebe and Dr T Mizuno. We are also thankful to Mr S Kimura, and Mmes K Sumiyoshi and C Nishida (Nanae Fish Culture Experimental Station) for their support. This study was supported in part by a Grant-in-Aid for Scientific Research (C) to EY (Nos 06760165 and 07660230) from the Ministry of Education, Science, Sports and Culture, Japan.

\section{REFERENCES}

1. Thorgaard GH. Chromosome set manipulation and sex control in fish. In: Hoar WS, Randall DJ, Donaldson EM, (eds). Fish Physiology, Vol. 9. Academic Press, New York. 1983; 405-434.

2. Arai $\mathrm{K}$. The current status of chromosome manipulation in aquaculture. Suisanzoshoku 2000; 45: 411-416.

3. Arai K. Genetic improvement aquaculture finfish species by chromosome manipulation techniques in Japan. Aquaculture 2001; 197: 205-228.

4. Wada K. Principle and technique in chromosome manipulation. 3: Invertebrate. In: Suzuki $\mathrm{R}$ (ed). Chromosome Manipulation and its Application for Aquaculture, Vol. 75. Suisangaku Series. Kouseisha Kouseikaku, Tokyo. 1989; 30-40.

5. Webb TA, Kowalski WJ, Fluck RA. Microtubule-based movements during ooplasmic segregation in the medaka fish egg (Oryzias latipes). Biol. Bull. 1995; 188: 146-156. 
6. Jesuthasan S, Strähle U. Dynamic microtubules and specification of the zebrafish embryonic axis. Curr: Biol. 1996; 7: 31-42.

7. Solnica-Krezel L, Driever W. Microtubule array of the zebrafish yolk cell: Organization and function during epiboly. Development 1994; 120: 2443-2455.

8. Yamaha E, Mizuno T, Hasebe Y, Takeda H, Yamazaki F. Dorsal specification in the blastoderm at the blastula stage in goldfish, Carassius auratus. Dev. Growth Differ. 1998; 40: 267-275.

9. Onozato $\mathrm{H}$, Yamaha E. Induction of gynogenesis with ultraviolet rays in four species of Salmoniformes. Nippon Suisan Gakkaishi 1983; 49: 693-699.

10. Nagoya $H$, Kimoto $T$, Onozato $H$. Diploid gynogenesis induced by suppression of the second or the third cleavage in the goldfish, Carassius auratus. Bull. Natl Res. Inst. Aquacult. 1990; 18: 1-6.

11. Mizuno T, Yamaha E, Yamazaki F. Localized axis determinant in the early cleavage embryo of the goldfish, Carassius auratus. Dev. Genes Evol. 1997; 206: 389396.

12. Stachel SE, Grunwald DJ, Myers PZ. Lithium perturbation and goosecoid expression identify dorsal specification pathway in the pregastrula zebrafish. Development 1993; 117: 1261-1274.
13. Kao KR, Elinson RP. The entire mesodermal mantle behaves as Spemann's organizer in dorsoanterior enhanced Xenopus laevis embryos. Dev. Biol. 1998; 127: 64-77.

14. Yamaha E, Usui K, Onozato $H$, Hamada $K$. A method for dechorionation in goldfish Carassius auratus. Nippon Suisan Gakkaishi 1986; 52: 1929-1934.

15. Yamaha E, Mizuno T, Matsushita K, Hasebe Y. Developmental staging in goldfish during the pre-gastrula stage. Nippon Suisan Gakkaishi 1999; 65: 709-717.

16. Katow $\mathrm{H}$. Observation of blastodisc formation by cytochalasin B in the zebrafish, Brachydanio rerio. Dev. Growth Differ: 1983; 25: 477-484.

17. Topczewski J, Solnica-Krezel L. Cytoskeletal dynamics of the zebrafish embryo. In: Detrich HW, Westerfield M, Zon LI (eds). The Zebrafish Biology: Methods in Cell Biology, Vo. 59. Academic Press, San Diego. 1999; 206-226.

18. Trinkaus JP. Mechanism of Fundulus epiboly - A current view. Am. Zool. 1984; 24: 673-688.

19. Mizuno T, Yamaha E, Kuroiwa T, Takeda H. Removal of vegetal yolk causes dorsal deficiencies and impairs dorsalinducing ability of the yolk cell in zebrafish. Mech. Dev. 1999; 81: $35-47$.

20. Trimble LM, Fluck RA. Indicators of the dorsoventral axis in medaka (Oryzias latipes) zygotes. Fish Biol. J. Medaka 1995; 7: 37-41. 